\title{
Long period fibre gratings photoinscribed in a microstructured polymer optical fibre by $\mathrm{UV}$ radiation
}

\author{
David Sáez-Rodríguez ${ }^{1}$, Jose L. Cruz Munoz ${ }^{1}$, Ian Johnson ${ }^{2}$, David J. Webb ${ }^{2}$, Maryanne C. J. \\ Large $^{3}$, Alexander Argyros ${ }^{3}$ \\ ${ }^{1}$ Dpto. de Fisica Aplicada y Electromagnetismo. Universidad de Valencia., Dr. Moliner 50, \\ Burjassot 46100, Valencia, Spain \\ ${ }^{2}$ Photonics Research Group Aston University, Aston Triangle Birmingham, B4 7ET, UK \\ ${ }^{3}$ Institute of Photonics and Optical Science, University of Sydney, NSW 2006 Australia
}

\begin{abstract}
Long period gratings were written step-by-step in microstructured poly(methyl methacrylate) (PMMA) fibre using a continuous wave $\mathrm{HeCd}$ laser at $325 \mathrm{~nm}$ irradiating the fibre with a power of $1 \mathrm{~mW}$. The grating had a length of $2 \mathrm{~cm}$ and a period of $1 \mathrm{~mm}$. A series of cladding mode coupling resonances were observed throughout the spectral region studied of 600 to $1100 \mathrm{~nm}$. The resonance wavelengths were shown to be sensitive to both surrounding refractive index and the water content of the polymer fibre.
\end{abstract}

Keywords: optical fibre sensor, long period grating, humidity, refractive index, mPOF, PCF

\section{INTRODUCTION}

Optical fibre Bragg and long period gratings have been studied for many years now and are well established sensing devices [1]; this applies particularly to fibre Bragg grating sensors which are supplied commercially by many companies around the world. Despite this apparent maturity, research in this field shows no sign of abating and one area we have been investigating recently is the recording of gratings in polymeric optical fibres. The motivation here is to be able to utilise the different material properties of polymers compared to the traditional silica fibre material. As an example relevant to strain monitoring applications, polymeric fibre can have a much smaller Young's modulus and much higher failure strain than silica fibre [2].

Long period gratings (LPGs) have previously been produced in microstructured polymer optical fibre (mPOF) by mechanical deformation coupled with heating [3]. In this paper we study, for the first time to our knowledge, the properties of LPGs produced using direct photoinscription using UV light.

\section{UV INSCRIPTION}

The fibre used in this work is made of polymethlymethacrylate (PMMA) with a refractive index of around 1.49. The outside diameter of fibre was $250 \mu \mathrm{m}$ while the fibre core was elliptical having a large diameter of $7.6 \mu \mathrm{m}$ and small diameter of $4.2 \mu \mathrm{m}$. The core was surrounded by 56 air holes with $0.72 \mu \mathrm{m}$ diameter and a separation of $2.59 \mu \mathrm{m}$, which gave a ratio of hole diameter to spacing of $\mathrm{d} / \Lambda=0.28$. As a consequence of the elliptical core the fibre has a nonnegligible birefringence.

Before writing the gratings, the ends of the MPOF fibre were cleaved using a razer balde with the fibre on a hot plate [4] and then spliced to two single mode silica fibres. The mPOF fibre was cleaved on a hot plate at $80^{\circ} \mathrm{C}$ in order to achieve a flat front surface and to prevent the air holes from collapse. The fibres to be spliced were mounted on XYZ stages in order to align their cores, the alignment being optimized by monitoring the light transmitted through the junction. The aligned fibres were fixed with Loctite 3525 glue cured during 10 minutes of exposure to a UV lamp having $0.5 \mathrm{~W}$ of nominal power. Finally, the splices were sleeved into silica tubes of $2.75 \mathrm{~mm}$ outer diameter in order to strengthen them. The insertion loss of the spliced fibre was measured to be $14 \mathrm{~dB}$ in the best case. Fig. 1 shows a SEM photograph of the cross section of the cleaved fibre, the elliptical core can be observed as well as a scratch made by the cleaver.

Photonic Crystal Fibers III, edited by Kyriacos Kalli, Proc. of SPIE Vol. 7357

73570L · C 2009 SPIE · CCC code: 0277-786X/09/\$18 · doi: 10.1117/12.823292

Proc. of SPIE Vol. 7357 73570L-1 


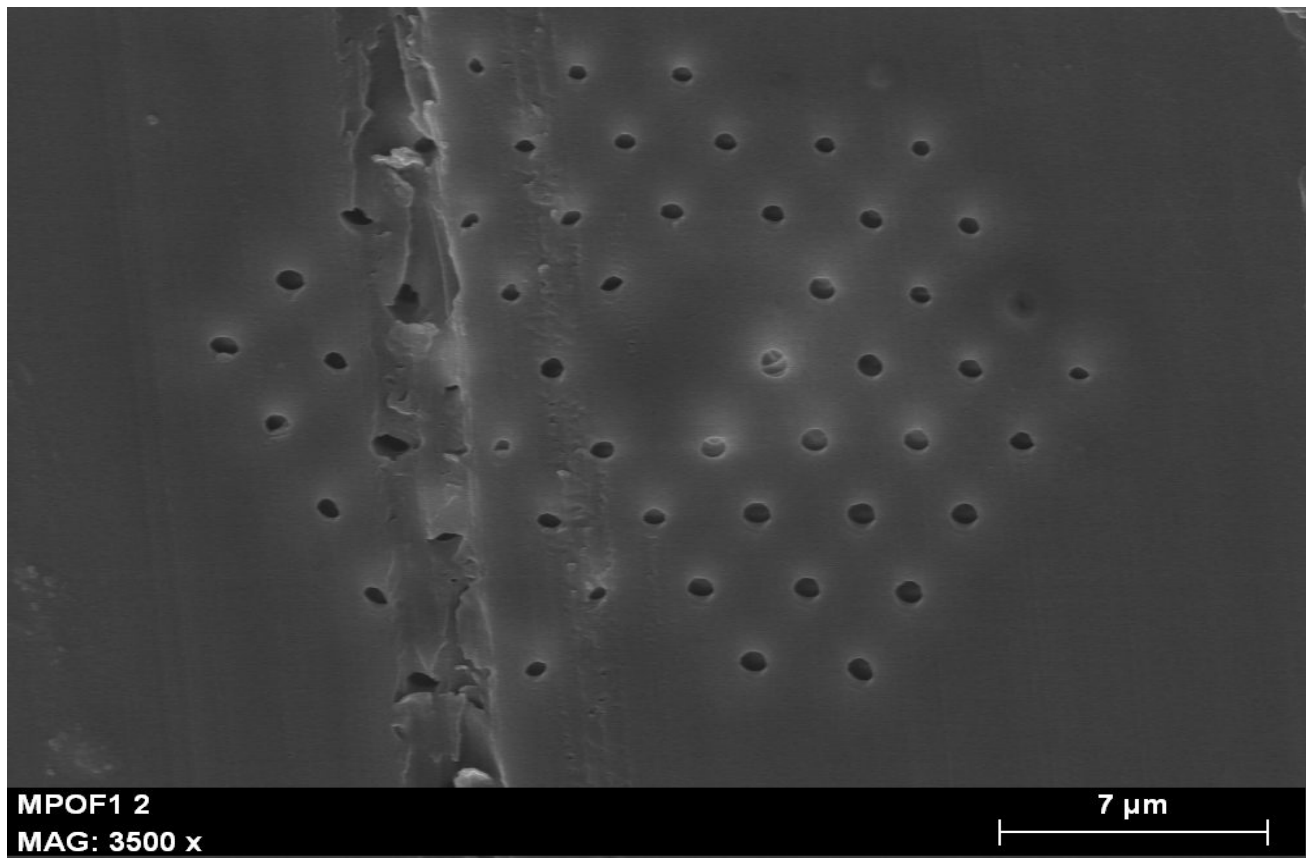

Fig. 1. Electron microscope photograph of a transversal cut of the mPOF.

The grating was written by irradiating the fibre with a continuous wave $\mathrm{He}-\mathrm{Cd}$ laser at a wavelength of $325 \mathrm{~nm}$. The laser delivers a maximum power of $30 \mathrm{~mW}$ and the beam has about a $1.2 \mathrm{~mm}$ diameter. The beam was focused onto the fibre by a spherical lens of $20 \mathrm{~cm}$ focal length. The mPOF was placed on two XYZ translation stages so that it could be aligned with the laser beam. Long period gratings were written step by step scanning the laser over the fibre and shutting the beam off each half period. The translation stage to scan the laser beam has a precision of $10 \mu \mathrm{m}$. The experimental setup is shown in the sketch of Fig. 2.

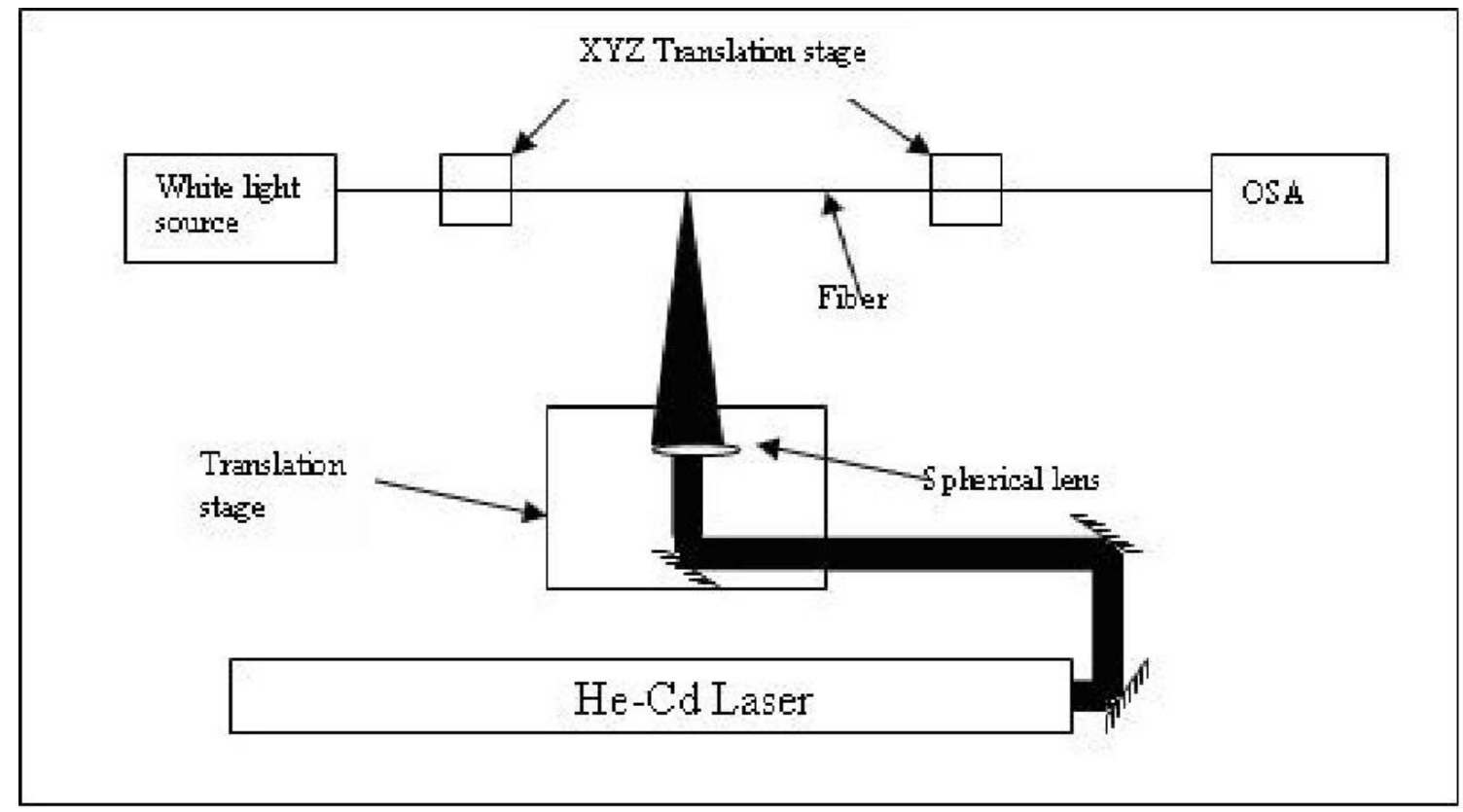

Fig 2. Experimental Setup 
When using the lens, the power delivered by this laser is big enough to melt the polymer when the beam is focused over the fibre. After several attempts we concluded that, to reach optimum index change in the fibre, the laser has to be operated at $1 \mathrm{~mW}$ output power and the beam has to be focused over the writing point for six seconds with interruptions of two seconds between exposures.

The long period grating fabricated was $2 \mathrm{~cm}$ in length and had a period of $1 \mathrm{~mm}$. The spectrum was measured using an optical spectrum analyser (OSA) with maximum resolution of $50 \mathrm{pm}$, wavelength range between $350 \mathrm{~nm}$ and $1750 \mathrm{~nm}$ and dynamic range of $90 \mathrm{dBm}$. A halogen lamp of $150 \mathrm{~W}$ was used as light source in the experiments. Fig. 3 shows the spectrum of the grating measured with $5 \mathrm{~nm}$ resolution in the wavelength range 600 to $1100 \mathrm{~nm}$. The spectrum has six notches corresponding to the coupling between the core guided mode and different cladding modes. The resonance at $950 \mathrm{~nm}$ is split into two bands that might correspond to two polarization states.

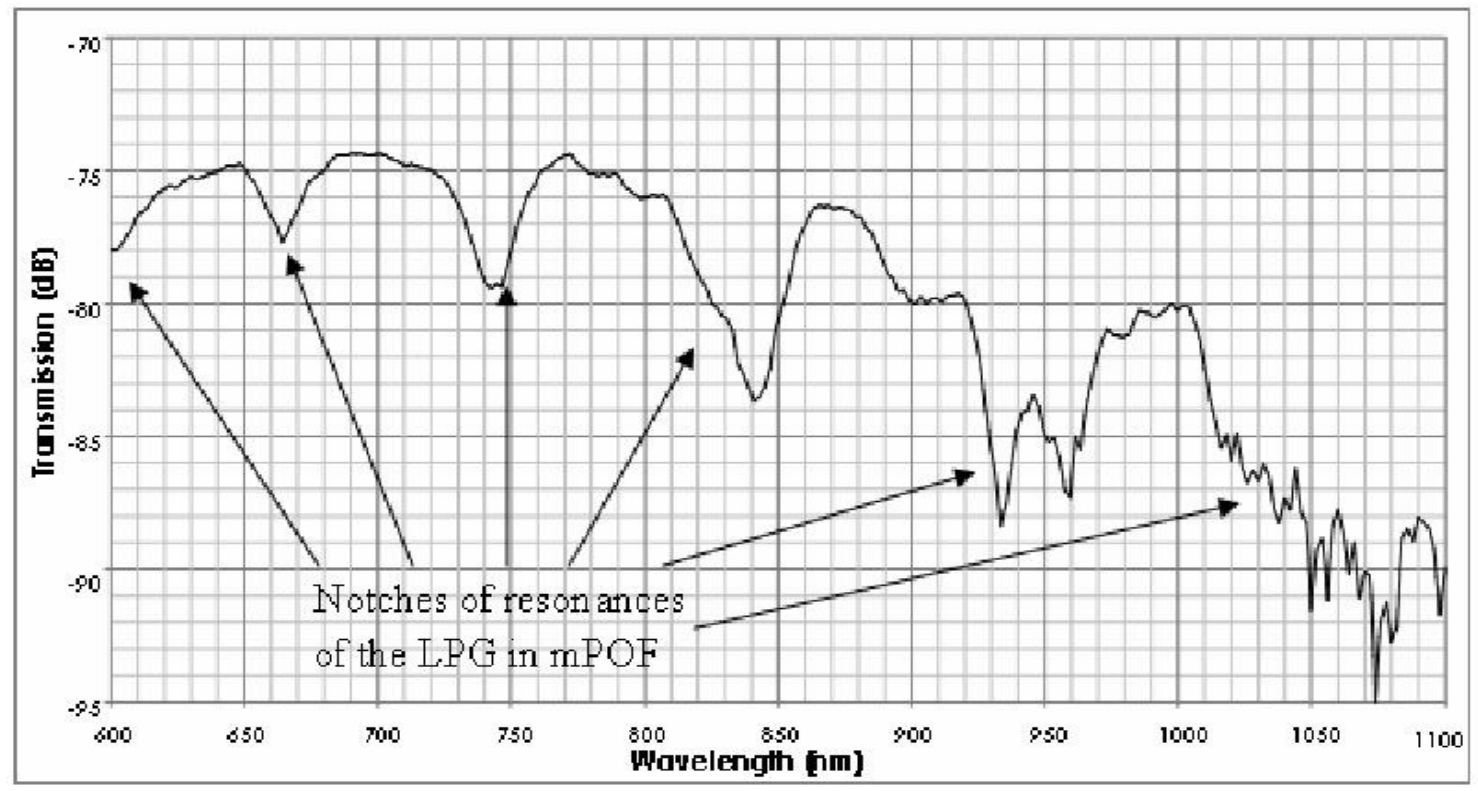

Fig. 3. Spectrum of LPG in mPOF.

\section{SPECTRAL RESPONSE WHEN EXPOSED TO WATER}

The response of the device to water is divided in two parts: the first one is the diffusion into the fibre, the second one is the drying process of the fibre. The MPOF grating was immersed in a bath of distilled water and the grating spectrum was measured for 8.5 hours until the diffusion of water in the fibre reached a stationary state. We show in Fig. 4 the wavelength shift of the resonance initially situated at $747 \mathrm{~nm}$ in Fig. 1.

The resonant wavelength $\lambda$ depends on the grating period $\Lambda$ and on the difference between the effective indices of the core and cladding modes $\left(\mathrm{n}_{\mathrm{co}}\right.$ and $\left.\mathrm{n}_{\mathrm{cl}}\right)$ through the expression:

$$
\lambda=\Lambda\left(\mathrm{n}_{\mathrm{co}}-\mathrm{n}_{\mathrm{cl}}\right)
$$

Care must be taken with this expression as the effective indices are both functions of wavelength [6]. Furthermore, unlike the usual situations where LPGs are used to sense temperature, strain, index, or bending, the measurand cannot be assumed to affect the core and cladding simultaneously. Instead, the cladding is affected initially as the water is absorbed, leading to the observed positive wavelength shift. As the cladding becomes saturated and the water penetrates to the core the wavelength starts to decrease, attaining a final steady state value with a net decrease in wavelength for this cladding mode. 
When the fibre is taken out of the water the polymer dries out and the opposite behaviour is observed. Fig. 5 shows the drying process. Initially the resonance shifts towards shorter wavelengths as water diffuses out of the cladding and afterwards it shifts upwards when the core starts drying. The spectrum was measured during 12 hours and it did not reach the stationary state, indicating that some moisture remains in the fibre for a long time. After drying out the fibre by gentle heating $\left(<45^{\circ} \mathrm{C}\right)$, the spectrum of Fig. 3 was recovered. Notice that the resonance under test was out of the wavelength measurement range during the time interval between 10 and 220 minutes.

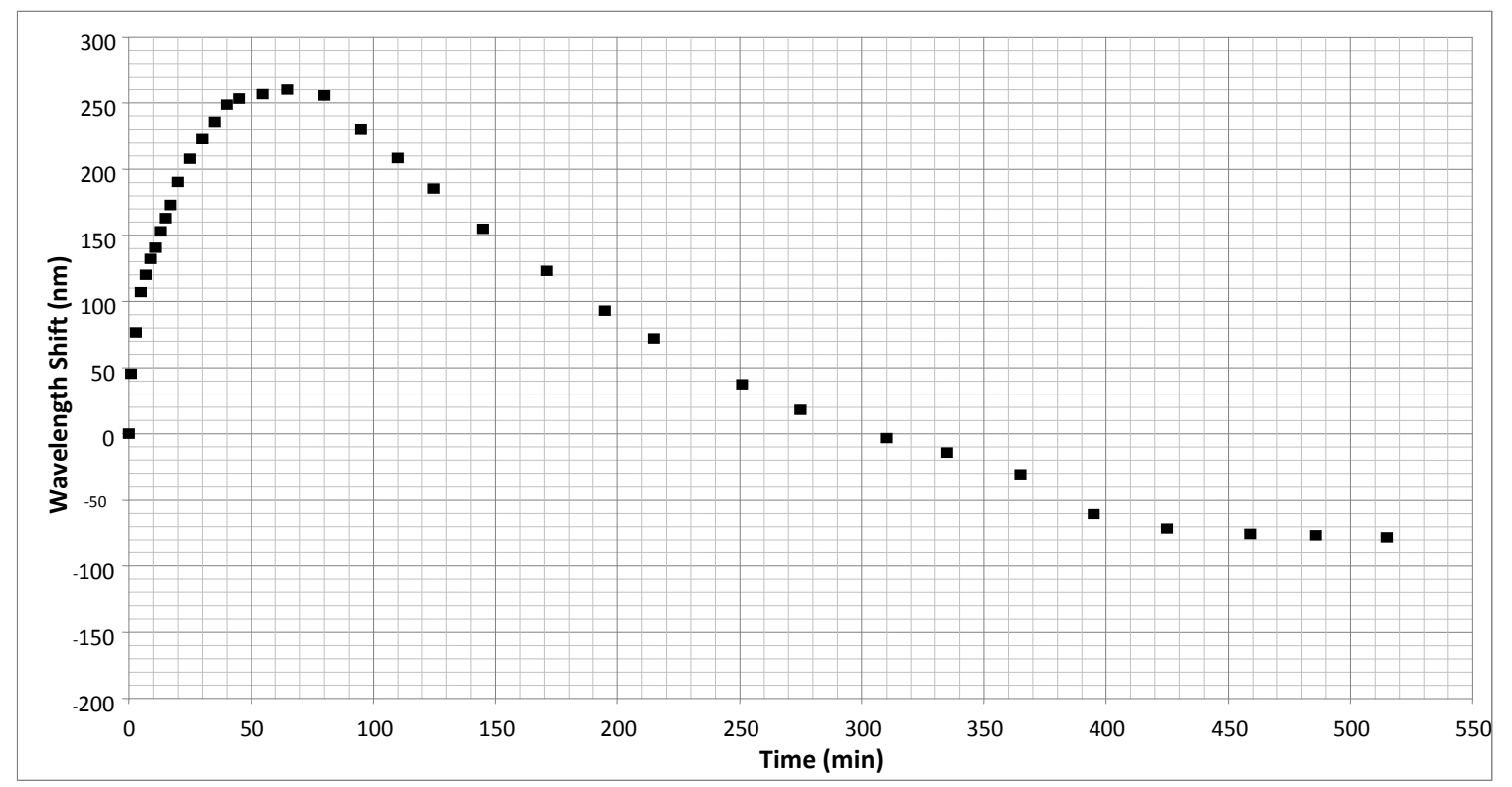

Fig. 4. Wavelength shift of a particular resonance $(747 \mathrm{~nm}$ in figure 3$)$ due to the diffusion of water into the fibre.

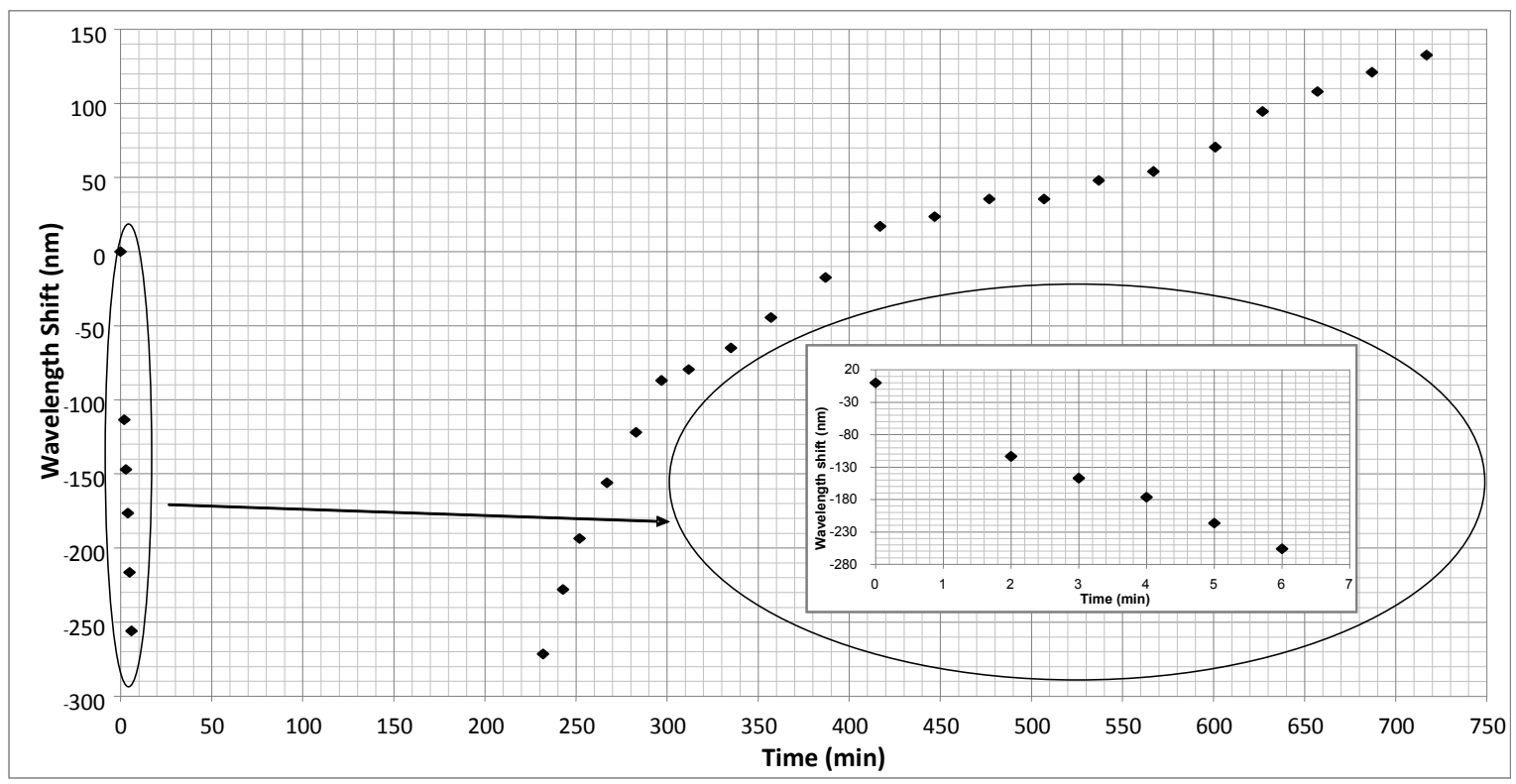

Fig. 5 Wavelength shift of a particular resonance (1070nm in figure 3) when the fibre dries out.

Some modes of the grating spectrum exhibit a double resonance within the measurement range, with the core mode coupling energy to a cladding mode at two different wavelengths $[5,6]$. These resonances shift in opposite spectral directions when the properties of the waveguide are modified. As an example, Fig. 6 shows the evolution of a double 
resonance while the fibre dries out: two resonances were measured at 600 and $1000 \mathrm{~nm} 19$ minutes after taking the fibre out of the water bath, these resonances move one towards the other and five minutes later they coincide at $820 \mathrm{~nm}$, afterwards, they gradually disappear.

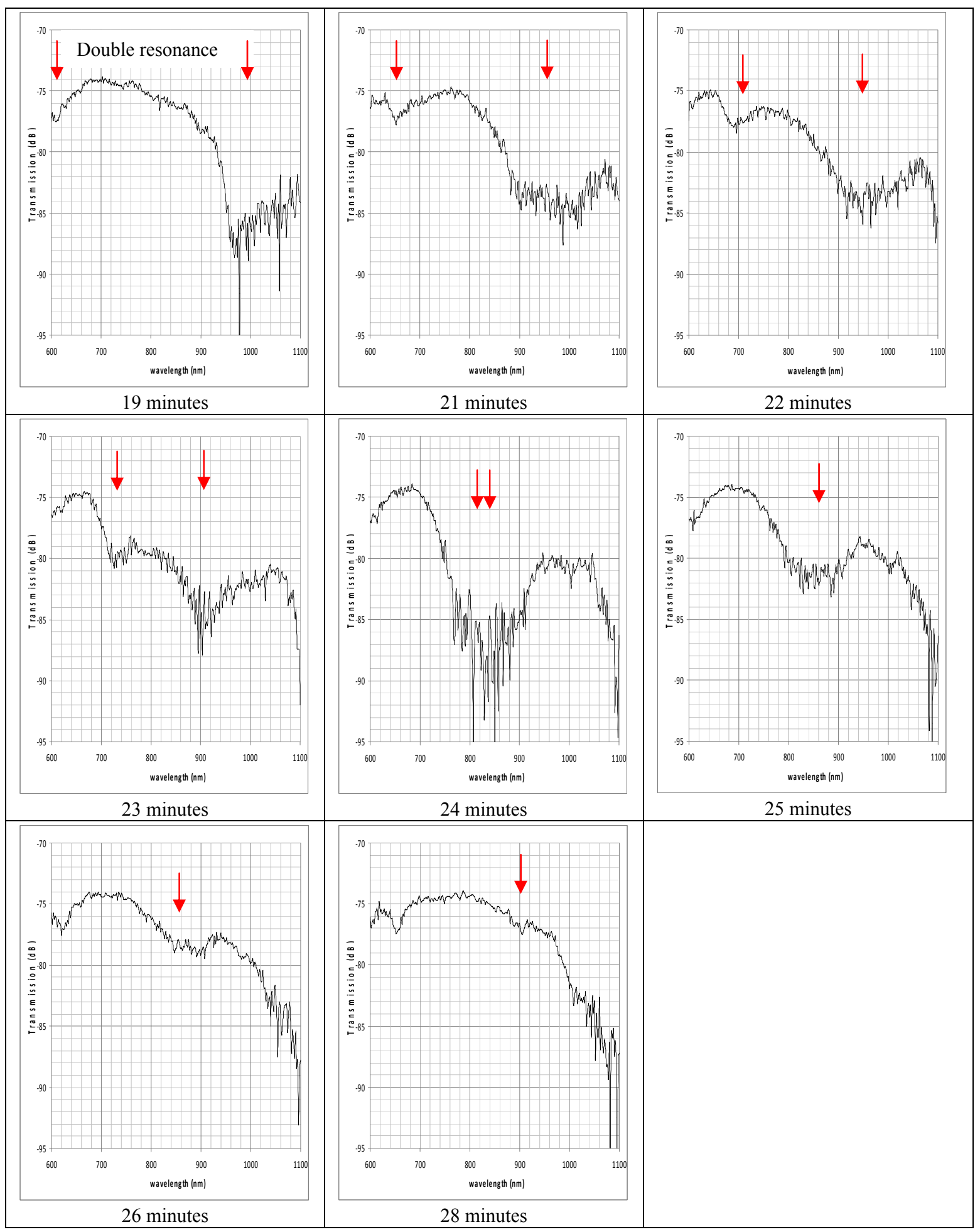

Fig. 6 Evolution of a double resonance as the fibre dries out. 


\section{SPECTRAL RESPONSE OF THE DEVICE TO THE REFRACTIVE INDEX OF THE SURROUNDING MEDIUM}

To measure the spectral response of the device to the refractive index of the surrounding medium we used a set of calibrated oils with refractive indices lying between 1.43 and 1.56. The liquids that we used for these experiments were provided by Cargille Laboratories[7] and are calibrated at $532 \mathrm{~nm}$ and $25^{\circ} \mathrm{C}$. The temperature coefficient of these liquids, provided by the manufacturer, is about $-4 \times 10^{-4} \mathrm{C}^{-1}$.

The variations of several resonances have been measured and are shown in Fig. 7. In the figures it can be observed that the wavelength shift is positive for small external indices, it has a maximum when the index of the oil is 1.49 and then it decreases for higher indices than 1.49. The refractive index of the polymer material is 1.49 so, when the surrounding oil matches this index the interface between oil and polymer effectively no longer exists and the outer waveguide is not defined giving rise to this divergent behaviour. The wavelength shift is positive for indices lower than 1.49 because the general sensitivity factor of the LPG, $\gamma$, defined in [6] is negative since $d \lambda_{\text {res }} / d \Lambda<0$ (where $\lambda_{\text {res }}$ is the wavelength resonance and $\Lambda$ is the period of LPG) for $\mathrm{mPOF}$ [3]. Moreover the depth of the notches remain almost constant until the external index approaches the index of the material (1.49), at this point the notches disappear and for indices larger than 1.49 the resonances appear again. Notice that silica fibres have a similar behaviour in the range of refractive index from 1 to $1.45[8]$.

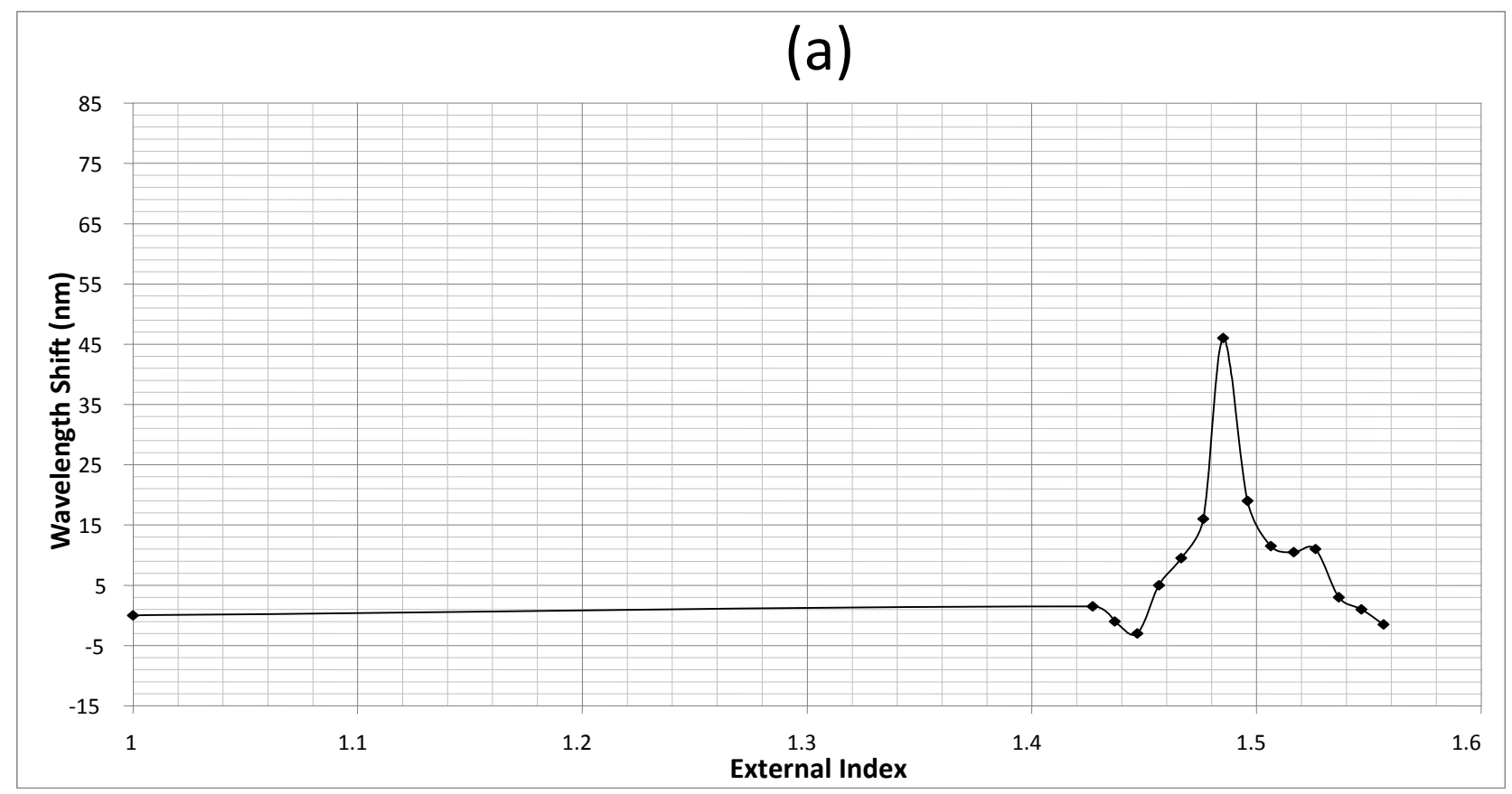




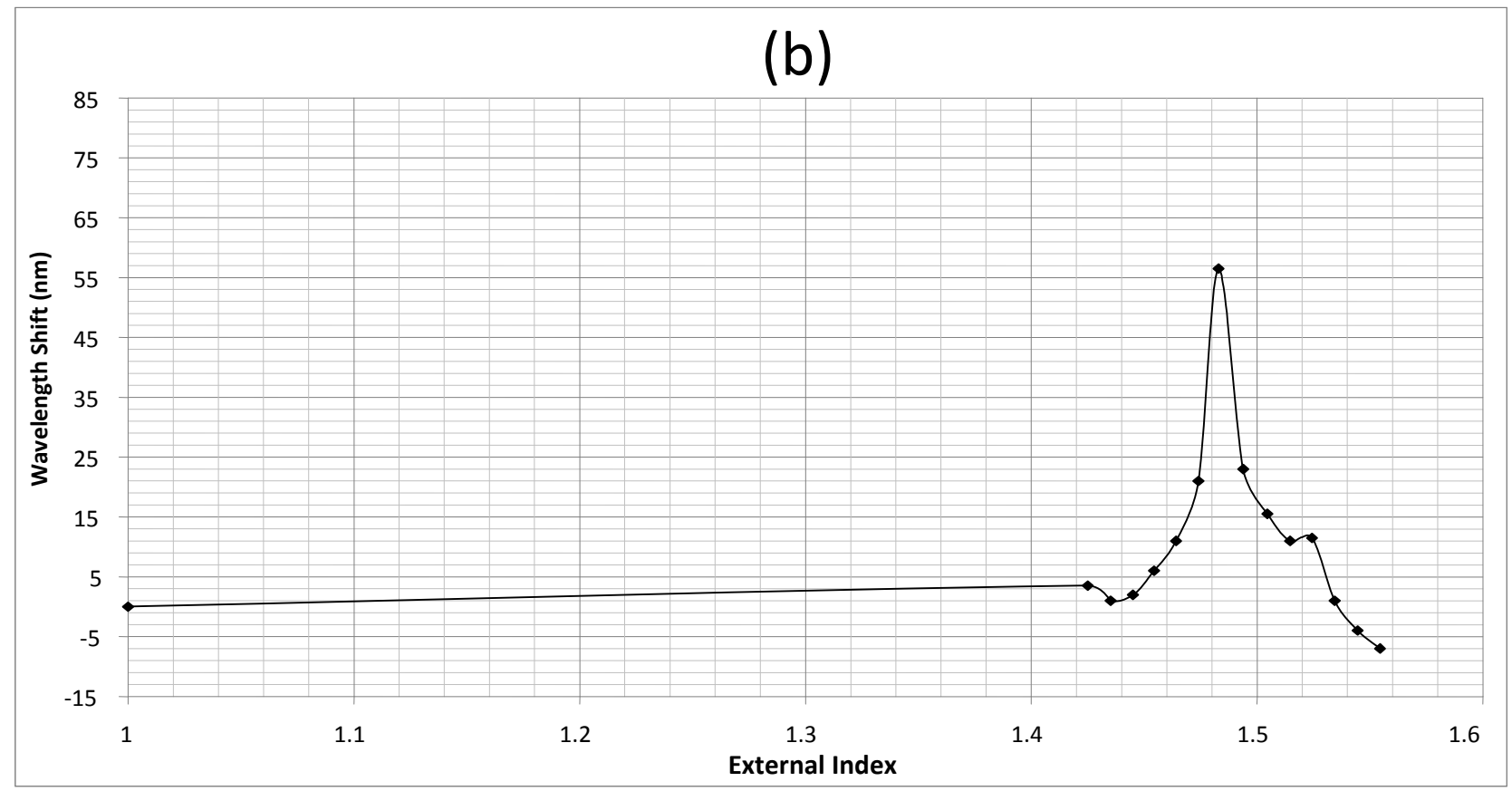

(c)

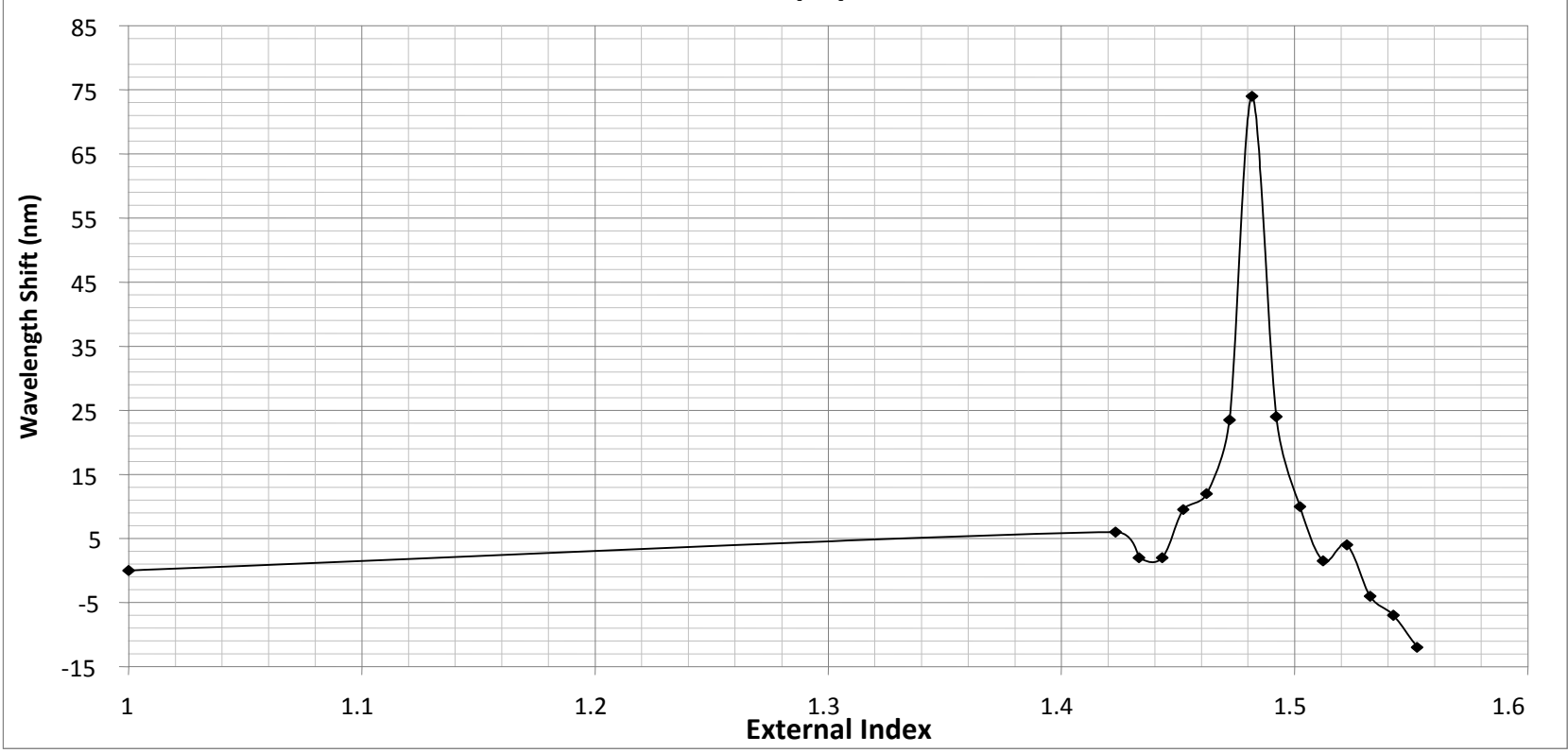

Fig. 7 Wavelength shift of several resonances as a function of the external refractive index:

(a) Initial resonance at $664.5 \mathrm{~nm}$. (b) Initial resonance at $747 \mathrm{~nm}$. (c) Initial resonance at $842.5 \mathrm{~nm}$. 


\section{CONCLUSION}

In this work we have demonstrated what is to our knowledge the first LPG photoinscribed in microstructured polymer optical fibre using UV radiation. Furthermore the response of the LPG to water and to the refractive index of the surrounding medium has been investigated. The diffusion of water into the fibre has been optically characterised and the device might work as a humidity sensor, though the response time for this fibre is quite slow. The spectral response to several oils surrounding the LPG has a maximum sensitivity for a refractive index of 1.49 (higher than silica), therefore it might be applied for detection of chemicals with refractive indices higher than silica glass.

\section{ACKNOWLEDGEMENTS}

This work has been supported by the Ministerio de Educación y Ciencia Español (TEC 2009-05490) and the UK Engineering and Physical Sciences Research Council. D. Sáez acknowledges assistance of FPU programmes.

\section{REFERENCES}

[1] Othonos, A. and Kalli, K., "Fiber Bragg Gratings: Fundamentals and Applications in Telecommunications and Sensing," Artech House, (1999).

[2]Aressy, M., "Manufacturing optimisation and mechanical properties of polymer optical fibre," MPhil Thesis, Birmingham University, UK, (2006).

[3]Hiscocks, M. P., van Eijkelenborg, M. A., Argyros, A. and Large, M. C. J., "Stable imprinting of long-period gratings in microstructured polymer optical fibre", Optics Express, 14(11), 4644-4649 (2006).

[4]Law, S. H., Harvey, J. D., Kruhlak, R. J., Song, M., Wu, E., Barton, G. W., van Eijkelenborg, M. A. and Large, M. C. J., "Cleaving of microstructured polymer optical fibres" Optics Communications, 258(2), 193-202 (2006 ).

[5] Shu, X., Zhu, X., Jiang, S., Shi, W. and Huang, D., "High sensitivity of dual resonant peaks of long-period fibre grating to surrounding refractive index changes", Electronics Letters, 35(18), 1580-1581 (1999).

[6]Shu, X., Zhang, L. and Bennion, I., "Sensitivity Characteristics of long-period fibre gratings”, Journal of Lightwave Technology, 20(2), 255-266 (2002).

[7] http://www.cargille.com/opticalintro.shtml

[8]Patrick, H. J., Kersey, A. D. and Bucholtz, F., "Analysis of the Response of Long Period Fibre Gratings to External Index of Refraction”, Journal of Lightwave Technology, 16(9), 1606-12 (1998). 